\title{
$\pi-\eta$ MIXING FROM QCD SUM RULES
}

\author{
Chuan-Tsung Chan ${ }^{(1)}$, E. M. Henley ${ }^{(1),(2)}$, and Th. Meissner ${ }^{(2)}$ \\ (1) Physics Department, FM-15, University of Washington, Seattle, WA 98195 \\ (2) Institute for Nuclear Theory, HN-12, University of Washington, Seattle, WA 98195
}

(March, 1994)

\begin{abstract}
The $q^{2}$ dependence of the $\pi-\eta$ mixing amplitude is examined with the use of QCD sum rules. The linear slope of the mixing function $\theta\left(q^{2}\right)$ is found to be much smaller than that for $\rho$ - $\omega$ mixing. Thus the mixing amplitude is approximately the same in the space-like region as in the time-like one, and one may neglect the $q^{2}$ dependence of the mixing. A comparison between a hadron-meson, an effective chiral model, and the QCD sum rules approaches is made.
\end{abstract}




\section{INTRODUCTION}

The recent interest in examining the off-shell behavior of $\rho$ - $\omega$ mixing [1] and $\pi-\eta$ mixing [5] is based on the observation of Goldman, Henderson and Thomas (GHT) [1] that the meson mixing previously used in the calculation of the nucleon-nucleon (N-N) charge-symmetry-violating (CSV) potential [8] may not be correct. In particular, the value of $\rho$ - $\omega$ mixing found experimentally in the time-like region $\left(q^{2}>0\right)$ may not apply in the space-like region, from which the CSV potential is generated. Subsequently, other authors have discussed this issue for $\rho$ - $\omega$ mixing by using different effective models [1, 3], 4], and also find appreciable $q^{2}$ dependence of the mixing amplitude. With the advent of QCD sum rules [9] and their phenomenological success in describing hadronic properties [10 12], it is natural to apply this technique to meson-mixing, particularly since the method is closer to QCD than other models that have been used.

In the pioneering work of Shifman, Vainshtein and Zakharov (SVZ) 9], the "on shell" $\rho-\omega$ mixing was used as an illustration of the power of QCD sum rules. However, in their paper some fine points remain elusive and there is no discussion of the "off-shell" behavior of meson-mixing. These details were re-examined by Hatsuda, Henley, Meissner and Krein (HHMK) [4], who used both Borel and finite-energy sum rules, together with dispersion relations to determine the momentum-dependence of $\rho-\omega$ mixing. They found a rapid $q^{2}$ variation of the mixing parameter $\theta\left(q^{2}\right)$. In this paper we shall follow the spirit of HHMK in applying it to $\pi-\eta$ mixing. By performing the Borel analysis, we can extract $\theta\left(q^{2}\right)$ as a function of the CSV parameters, and we can also study how the QCD condensates affect the mixing function $\theta\left(q^{2}\right)$.

This paper is organized as follows: In section I, we establish notations and definitions. The calculations are described in section III. In section IV, we relate our results to those

of GHT [1] and Piekarewicz-Williams (PW) [3]. Finally, in section V, conclusions and a summary are presented.

\section{II. $\pi-\eta$ MIXING (FORMALISM)}

The $\pi-\eta$ mixing function $\theta\left(q^{2}\right)$ is defined by the mixed correlator of $\pi^{0}$ and $\eta^{0}$ 


$$
\pi^{\pi \eta}\left(q^{2}\right)=i \int d^{4} x e^{i q x}<T \pi^{0}(x) \eta^{0}(0)>
$$

where $\pi^{0}$ and $\eta^{0}$ are eigenstates of an isosymmetric strong-interaction Hamiltonian.

We can express the physical $\pi-\eta$ fields, which are eigenstates of the full strong interaction Hamiltonian, in terms of $\pi^{0}, \eta^{0}$, by introducing the mixing matrix

$$
\left(\begin{array}{l}
\pi \\
\eta
\end{array}\right)=\left(\begin{array}{cc}
1 & \epsilon \\
-\epsilon & 1
\end{array}\right)\left(\begin{array}{l}
\pi^{0} \\
\eta^{0}
\end{array}\right),
$$

where $\epsilon$ is a "mixing angle". By saturating the $I m \pi^{\pi \eta}$ with the $\pi$ and $\eta$ poles and using a dispersion relation for $\operatorname{Re} \pi^{\pi \eta}\left(q^{2}\right)$, we can write

$$
\pi^{\pi \eta}\left(q^{2}\right) \equiv \frac{\theta\left(q^{2}\right)}{\left(q^{2}-m_{\pi}^{2}+i \epsilon\right)\left(q^{2}-m_{\eta}^{2}+i \epsilon\right)} .
$$

where the mixing function is related to the mixing angle $\epsilon$ as

$$
\theta\left(q^{2}\right)=\left[\epsilon\left(m_{\eta}^{2}\right)-\epsilon\left(m_{\pi}^{2}\right)\right] q^{2}+\left[m_{\eta}^{2} \epsilon\left(m_{\pi}^{2}\right)-m_{\pi}^{2} \epsilon\left(m_{\eta}^{2}\right)\right]
$$

Since the $\epsilon$ "mixing angle" may be a function of $q^{2}$, the value of $\epsilon\left(m_{\pi}^{2}\right)$ may be different from that of $\epsilon\left(m_{\eta}^{2}\right)$; in this way the mixing function $\theta\left(q^{2}\right)$ develops a linear dependence on $q^{2}$.

On the other hand, in order to study the meson properties from the quark-gluon degrees of freedom without being plagued by the problem of wave functions, we need to introduce interpolating quark currents for the correlation function, and the meson properties are represented by the experimentally measurable matrix elements of these quark currents. In the present case, we choose to work with the axial vector currents rather than pseudoscalar ones for a number of reasons. Foremost is that, although $\pi$ and $\eta$ are pseudoscalar particles, the use of the axial vector currents gives a better convergence property [9] 12]. Secondly, on the mass shell, the two currents are directly related. In an SU(3) notation, we use

$$
\begin{aligned}
j_{\mu}^{3}(x) & \equiv \frac{1}{2}\left(\bar{u} \gamma_{\mu} \gamma_{5} u(x)-\bar{d} \gamma_{\mu} \gamma_{5} d(x)\right) \\
j_{\nu}^{8}(x) & \equiv \frac{1}{2 \sqrt{3}}\left(\bar{u} \gamma_{\mu} \gamma_{5} u(x)+\bar{d} \gamma_{\mu} \gamma_{5} d(x)-2 \bar{s} \gamma_{\mu} \gamma_{5} s(x)\right)
\end{aligned}
$$

The correlator of these two currents is defined by

$$
\pi_{\mu \nu}^{\pi \eta} \equiv i \int d^{4} x e^{i q x}<T j_{\mu}^{3}(x) j_{\nu}^{8}(0)>
$$


Using the definition of the mixing angle $\epsilon,($ Eq. (3)), the PCAC relation for $\pi$ and $\eta$, $<0\left|j_{\mu}^{3}(0)\right| \pi(p)>=i f_{\pi} p_{\mu},<0\left|j_{\mu}^{8}(0)\right| \eta(p)>=i f_{\eta} p_{\mu}$, where $f_{\pi}$ and $f_{\eta}$ are the decay constants for $\pi$ and $\eta$, and the pole approximation for $\operatorname{Im} \pi_{\mu \nu}^{\pi \eta}$, we have:

$$
\frac{1}{\pi} \operatorname{Im} \pi_{\mu \nu}^{\pi \eta}=q_{\mu} q_{\nu} f_{\pi} \cdot f_{\eta}\left[\epsilon\left(m_{\pi}^{2}\right) \delta\left(q^{2}-m_{\pi}^{2}\right)-\epsilon\left(m_{\eta}^{2}\right) \delta\left(q^{2}-m_{\eta}^{2}\right)\right] .
$$

Because of the structure of PCAC and the $\pi-\eta$ pole approximation, there is no $q^{2} g_{\mu \nu}$ term in $\operatorname{Im} \pi_{\mu \nu}^{\pi \eta}$. In addition to the $\pi$ and $\eta$ poles, let us also include the next resonances in the $\operatorname{Im} \pi_{\mu \nu}^{\pi \eta}$; these resonances are a pair of pseudo-vector particles the $b_{1}(1235)$ (denoted by A) and $h_{1}(1170)$ (denoted by B); these resonances contribute to $\operatorname{Im} \pi_{\mu \nu}^{\pi \eta}$,

$$
\left(q_{\mu} q_{\nu}-q^{2} q_{\mu \nu}\right) \tilde{f}_{A} \tilde{f}_{B}\left[\epsilon^{\prime}\left(m_{A}^{2}\right) \delta\left(q^{2}-m_{A}^{2}\right)-\epsilon\left(m_{B}^{2}\right) \delta\left(q^{2}-m_{B}^{2}\right)\right]
$$

where $\tilde{f}_{A}, \tilde{f}_{B}$ are the decay constants for A and B particles.

Combining these two contributions, we can rewrite

$$
\begin{aligned}
\frac{1}{\pi} \operatorname{Im} \pi_{\mu \nu}^{\pi \eta}= & q_{\mu} q_{\nu} \operatorname{Im} \pi_{1}\left(q^{2}\right)-q^{2} g_{\mu \nu} \operatorname{Im} \pi_{2}\left(q^{2}\right) \\
\operatorname{Im} \pi_{1}\left(q^{2}\right) \equiv & g_{\pi} \delta\left(q^{2}-m_{\pi}^{2}\right)-g_{\eta} \delta\left(q^{2}-m_{\eta}^{2}\right) \\
& +h_{A} \delta\left(q^{2}-m_{A}^{2}\right)-h_{B} \delta\left(q^{2}-m_{B}^{2}\right) \\
& + \text { higher resonances }+ \text { continuum } \\
\operatorname{Im}_{2}\left(q^{2}\right)= & h_{A} \delta\left(q^{2}-m_{A}^{2}\right)-h_{B} \delta\left(q^{2}-m_{B}^{2}\right)+\text { higher resonances }+ \text { continuum },
\end{aligned}
$$

where

$$
\begin{array}{rlrl}
g_{\pi} & \equiv f_{\pi} f_{\eta} \epsilon\left(m_{\pi}^{2}\right) & g_{\eta} & \equiv f_{\pi} f_{\eta} \epsilon\left(m_{\eta}^{2}\right) \\
h_{A} \equiv \frac{\tilde{f}_{A} \tilde{f}_{B}}{m_{A}^{2}} \epsilon^{\prime}\left(m_{A}^{2}\right) & h_{B}=\frac{\tilde{f}_{A} \tilde{f}_{B}}{m_{B}^{2}} \epsilon^{\prime}\left(m_{B}^{2}\right)
\end{array}
$$

These parameters $g_{\pi}, g_{\eta}, h_{A}$ and $h_{B}$ are unknown and will be determined from the QCD sum rules. Once they are determined in terms of the CSV parameters and the Wilson coefficients of the OPE for the quark current-current correlation function, we can substitute these numbers into the mixing function $\theta\left(q^{2}\right)$ and discuss its momentum dependence in the region of interest.

In the case of $\pi-\eta$ mixing, in contrast to the case of $\rho-\omega$ mixing, there is no "on shell" value for $\theta\left(q^{2}\right)$, due to the large difference of $\pi$ and $\eta$ masses, $\Delta m \equiv m_{\eta}-m_{\pi}=412$ 
$\mathrm{MeV} \sim 3 m_{\pi}$; this mass difference presumably comes primarily from the s-quark contribution in the $\eta$. There is, thus, no direct experimental method to study $\pi-\eta$ mixing, unlike $e^{+} e^{-} \rightarrow \pi^{+} \pi^{-}$at the $\omega$-resonance [13,14, since $\pi$ and $\eta$ cannot be formed from one photon states in $e^{+} e^{-}$collisions; however, the decay of the $\eta^{0}$ to $\pi^{+} \pi^{-} \pi^{0}$ via the $\pi^{0}$ pole serves to set an "experimental" mixing at the $\eta$ mass [15]. Here we shall content ourselves to use experimentally measured masses for the $\pi(135), \eta(547), \mathrm{b}(1235)$ and $h_{1}(1170)$ particles as input parameters, and distinguish between $\epsilon\left(m_{\pi}^{2}\right), \epsilon\left(m_{\eta}^{2}\right), \epsilon^{\prime}\left(m_{A}^{2}\right)$, and $\epsilon^{\prime}\left(m_{B}^{2}\right)$ as independent variables. Although we do not rely on an expansion in terms of the mass difference $\delta m^{2} \equiv m_{\eta}^{2}-m_{\pi}^{2}$ to perform the calculations as in the $\rho$ - $\omega$ case, the large mass difference of $\pi$ and $\eta$ is comfortably accommodated without difficulty.

\section{CALCULATIONS}

One important ingredient of the QCD sum rules is the operator product expansion (OPE) 16] for the correlation functions. In our case, we calculate the OPE of $\pi_{\mu \nu}^{\pi \eta}$ by keeping operators up to dimension 6. For the Wilson coefficients, we work to first order in $\alpha_{s}, \alpha$ and $\frac{m q}{Q}$, where $\alpha_{s}(\alpha)$ is the fine structure constant for the strong (electromagnetic) interaction, and $m_{q}$ is the current mass of quark $q\left(m_{u}\right.$ for $u$ quark, and $m_{d}$ for $d$ quark). We use the definition $Q^{2}=-q^{2}$; we do not include the $q^{2}$ dependence of $\alpha_{s}$ but choose its value at 1 $\mathrm{GeV}^{2}, \alpha_{s}\left(1 \mathrm{GeV}^{2}\right) \sim 0.5$. Our calculation is similar to that for the $\rho-\omega$ case but we are dealing with axial vector currents for pseudoscalar mesons; thus, the reader may convince himself or herself of the signs of our results by counting the number of $\gamma$ matrices in each diagram. In the following, we shall neglect a detailed discussion of each diagram (c.f. ref. [4]) and simply write down the relevant answers:

$$
\begin{aligned}
\pi_{\mu \nu}^{\pi \eta}(q) & \equiv q_{\mu} q_{\nu} \pi_{1}\left(q^{2}\right)-q^{2} g_{\mu \nu} \pi_{2}\left(q^{2}\right), \quad Q^{2} \equiv-q^{2} \\
4 \sqrt{3} \pi_{1}\left(q^{2}\right) & \equiv C_{0} \ln Q^{2}+\frac{C_{1}}{Q^{2}}+\frac{C_{2}}{Q^{4}}+\frac{C_{3}}{Q^{6}}+O\left(\frac{1}{Q^{8}}\right) \\
4 \sqrt{3} \pi_{2}\left(q^{2}\right) & \equiv C_{0} \ln Q^{2}+\frac{C_{1}}{Q^{2}}-\frac{C_{2}}{Q^{4}}+\frac{C_{3}}{Q^{6}}+O\left(\frac{1}{Q^{8}}\right)
\end{aligned}
$$


where

$$
\begin{aligned}
C_{0}= & -\frac{\alpha}{16 \pi^{3}}+O\left(\alpha_{s}^{2}, \alpha^{2}\right), \quad \alpha_{s} \equiv \frac{g^{2}}{4 \pi}, \alpha \equiv \frac{e^{2}}{4 \pi} \\
C_{1}= & 0+O\left(m_{q}^{2}\right) \\
C_{2}= & 2\left[m_{u}<\bar{u} u>-m_{d}<\bar{d} d>\right] \\
C_{3}= & \frac{352}{81} \pi \alpha_{s}\left[<\bar{u} u>^{2}-<\bar{d} d>^{2}\right] \\
& +\frac{88}{243} \pi \alpha\left[4<\bar{u} u>^{2}-<\bar{d} d>^{2}\right] .
\end{aligned}
$$

It is helpful to note three points:

(1) For vector mesons, the conservation of the vector current leads to a $\pi_{\mu \nu}$ that must be proportional to $q_{\mu} q_{\nu}-q^{2} g_{\mu \nu}$; therefore $\pi_{1}=\pi_{2}$. This is no longer true in the $\pi-\eta$ case, since the axial vector current is not conserved. A side benefit is that we now have two independent sum rules for $\pi_{\mu \nu}^{\pi \eta}$, and we can take advantage of this property to extract information on the parameters appearing in the mixing function $\theta\left(q^{2}\right)$

(2) If we restrict ourselves to operators up to dimension 6 and make the vacuum saturation assumption (VSA) for the four quark condensate [9], we find that the $s$ quark in the $\eta$ current (Eq. (5)) does not contribute at all. The reason is that the $s$ quark could only arise in the four quark condensate $<\bar{q} \Gamma q \bar{s} \Gamma s>(q=$ up or down quark) which vanishes under the VSA.

(3) Taking the difference of the sum rules for $\pi_{1}$ and $\pi_{2}$ we see that the coefficient $C_{3}$ for the dimension 6 , four-quark condensate drops out. Therefore the uncertainty in this coefficient due to the assumption of the VSA can be ignored.

The next step is to relate these CSV parameters and condensates to the phenomenological parameters $g_{\pi}, g_{\eta}, h_{A}$ and $h_{B}$ in the RHS of $\operatorname{Im} \pi_{\mu \nu}$ (our model for $\operatorname{Im} \pi_{\mu \nu}$ ). In order to assure convergence, to accentuate the lower resonances and to lessen the effect of the continuum, we follow the standard practice of applying a Borel transformation to $\pi_{1}$ and $\pi_{2}$ [9],

$$
\hat{L}_{M}\left[f\left(Q^{2}\right)\right] \equiv \lim _{\substack{n \rightarrow \infty \\ Q^{2} \rightarrow \infty \\ \frac{Q^{2}}{n}=M^{2} \\ \text { fixed }}} \frac{1}{(n-1) !}\left(Q^{2}\right)^{n}\left(-\frac{d}{d Q^{2}}\right)^{n} .
$$

In this way, we obtain a set of simultaneously linear equations for $g_{\pi}, g_{\eta}, h_{A}$ and $h_{B}$ : 
The sum rule for $\pi_{1}$ gives

$$
\begin{aligned}
-C_{0}+C_{2}\left(\frac{1}{M^{4}}\right)+ & \frac{1}{2} C_{3}\left(\frac{1}{M^{6}}\right) \\
= & \frac{4 \sqrt{3}}{M^{2}}\left[g_{\pi} e^{-\frac{m_{\pi}^{2}}{M^{2}}}-g_{\eta} e^{-\frac{m_{\eta}^{2}}{M^{2}}}+h_{A} e^{-\frac{m_{A}^{2}}{M^{2}}}-h_{B} e^{-\frac{M_{B}^{2}}{M^{2}}}\right] \\
& + \text { higher resonances }\left(\pi^{\prime}, \eta^{\prime}\right)\left(A^{\prime}, B^{\prime}\right)+\text { continuum }
\end{aligned}
$$

and that for $\pi_{2}$ yields

$$
\begin{aligned}
-C_{0}-C_{2}\left(\frac{1}{M^{4}}\right) & +\frac{C_{3}}{2}\left(\frac{1}{M^{6}}\right) \\
& =\frac{4 \sqrt{3}}{M^{2}}\left[h_{A} e^{-\frac{m_{A}^{2}}{M^{2}}}-h_{B} e^{-\frac{m_{B}^{2}}{M^{2}}}\right]+\text { higher resonances }\left(A^{\prime}, B^{\prime}\right)+\text { continuum . }
\end{aligned}
$$

Taking the difference, we get

$$
2 C_{2}\left(\frac{1}{M^{4}}\right)=\frac{4 \sqrt{3}}{M^{2}}\left[g_{\pi} e^{-\frac{m_{\pi}^{2}}{M^{2}}}-g_{\eta} e^{-\frac{m_{\eta}^{2}}{M^{2}}}\right]+\text { higher resonances }\left(\pi^{\prime}, \eta^{\prime}\right) .
$$

It is worthwhile to call attention to the fact that the contributions of higher resonances $b_{1}(1235)$ and $h_{1}(1170)$ and continuum have been completely canceled in Eq. (16). The same is true for the electromagnetic continuum, whereas higher resonances in the pseudoscalar channel, such as $\pi^{\prime}(1300)-\eta^{\prime}(1290)$, will survive (in principle) in Eq. (16). However, it will turn out that taking these resonances explicitly into account does not change the Borel analysis noticeably. This is in contrast to the case of $\rho-\omega$ mixing where the corresponding high resonances $\left(\rho^{\prime}-\omega^{\prime}\right)$ are important.

By taking the derivative with respect to $\left(\frac{1}{M^{2}}\right)$ on both sides of Eq. (16), we obtain another sum rule:

$$
\frac{-C_{2}}{2 \sqrt{3}}=m_{\pi}^{2} g_{\pi} e^{-\frac{m_{\pi}^{2}}{M^{2}}}-m_{\eta}^{2} g_{\eta} e^{-\frac{m_{\eta}^{2}}{M^{2}}} .
$$

With these two equations, we can solve for $g_{\pi}$ and $g_{\eta}$ :

$$
\begin{aligned}
& g_{\pi}=\left(\frac{C_{2}}{2 \sqrt{3} M^{2}}\right) e^{m_{\pi}^{2} / M^{2}}\left(\frac{M^{2}+m_{\eta}^{2}}{m_{\eta}^{2}-m_{\pi}^{2}}\right) \\
& g_{\eta}=\left(\frac{C_{2}}{2 \sqrt{3} M^{2}}\right) e^{m_{\eta}^{2} / M^{2}}\left(\frac{M^{2}+m_{\pi}^{2}}{m_{\eta}^{2}-m_{\pi}^{2}}\right)
\end{aligned}
$$

Finally, the mixing function is

$$
\theta\left(q^{2}\right) \equiv \alpha\left(M^{2}\right) q^{2}+\beta\left(M^{2}\right)
$$


where

$$
\begin{aligned}
\alpha\left(M^{2}\right) & \equiv \frac{g_{\eta}-g_{\pi}}{f_{\pi}^{2}} \\
\beta\left(M^{2}\right) & \equiv \frac{m_{\eta}^{2} g_{\pi}-m_{\pi}^{2} g_{\eta}}{f_{\pi}^{2}} .
\end{aligned}
$$

where we assume the $\mathrm{SU}(3)$ symmetry for the decay constants $f_{\pi}=f_{\eta}$.

In Fig. 1 we plot the dependence of $g_{\pi}$ and $g_{\eta}$ on $M^{2}$. In the preferred region of 1.0 $\mathrm{GeV}^{2} \lesssim M \lesssim 1.6 \mathrm{GeV}^{2}$, the difference between $g_{\pi}$ and $g_{\eta}$ is small. Furthermore, it can be

seen from Fig. 1 and Eq. (18) that if $M^{2} \gg m_{\eta}^{2}, g_{\pi} \approx g_{\eta}$. We thus find that $\theta\left(q^{2}\right)$ has a weak $q^{2}$ dependence.

In order to fix the values of $g_{\pi}, g_{\eta}, \alpha$ and $\beta$, we choose a suitable Borel window and average the functions $g_{\pi}\left(M^{2}\right), g_{\eta}\left(M^{2}\right)$ over the range of $M^{2}\left(1 \mathrm{GeV}^{2} \sim 1.5 \mathrm{GeV}^{2}\right)$. At this point, one should note that if we included the higher resonances $\pi(1300)$ and $\eta(1295)$ in the phenomenological side of the sum rules and left their masses as parameters, we could perform the stability analysis to obtain the "optimal" values for $g_{\pi}$ and $g_{\eta}$. However, our numerical results do not suggest such "optimal" values are well-defined. The numerical values we obtain for these parameters are

$$
\begin{aligned}
& g_{\pi}=114 \mathrm{MeV}^{2} \quad g_{\eta}=117 \mathrm{MeV}^{2} \\
& \alpha=3.65 \times 10^{-4} \quad \beta=3660 \mathrm{MeV}^{2}
\end{aligned}
$$

All numbers have an error of about $10-20 \%$, which is due to the uncertainty of the isospin symmetry violating quantities $\frac{m_{d}-m_{u}}{m_{d}+m_{u}}$ and $\gamma=\frac{<\bar{d} d>}{<\bar{u} u>}-1$ in the coefficient $C_{2}$ and the fact that $M^{2} \gtrsim 0.9 \mathrm{GeV}^{2}$ for stability.

\section{COMPARISONS WITH OTHER MODELS}

In this section, we shall compare our results with those of other authors, who use a different approach.

$\pi-\eta$ mixing has been considered previously by Maltman and Goldman (MG) [5, [6] and Piekarewicz (P) [7]. Maltman used chiral perturbation theory [5] to calculate the $\pi$ - $\eta$ mixing to the one-loop order. The mixing function $\theta\left(q^{2}\right)$ is expressed in terms of meson masses and other physical observables; theoretical uncertainty comes from the electromagnetic mass 
difference of kaons. In order to verify the assumption which underlies the GHT calculation. Maltman and Goldman [6] also use a chiral quark model to calculate $\pi-\eta$ mixing and get a consistent result with chiral perturbation theory.

Piekarewicz [7] used a purely hadronic model to calculate $\pi-\eta$ mixing, where the mixing is generated by a $N \bar{N}$ loop. In order to fix the renormalization point for the divergent integral, he chose the "on shell" value of $m_{\eta}$ to fix the intercept of the mixing function.

He also derived a ratio between the slope at the origin of the $\rho$ - $\omega$ and $\pi-\eta$ mixing amplitudes

$$
\frac{<\pi|H| \eta>\text { slope at } q^{2}=0}{<\rho|H| \omega>\text { slope at } q^{2}=0} \simeq \frac{g_{\pi} g_{\eta}}{f_{\rho} g_{\omega}}
$$

which he claimed to be less model-dependent and to hold approximately for a reasonable range of $q^{2}$.

In view of the lack of "on-shell" experimental value for the $\pi-\eta$ mixing amplitude (the previous calculations are based on $\mathrm{SU}(3)$ mass splitting [17,18 of pseudoscalar mesons and a pole model analysis of the $\eta-\eta^{\prime}$ system [17,[18]), our calculation provides an independent result for the slope and intercept for the mixing function. We list the different results from the three calculations in Table 1.

It is to be noticed that our slopes are three times smaller than that of chiral perturbation theory and correspondingly so is the ratio $\alpha / \beta$. Because of the small slope, our mixing function only changes about $5 \%$ from $q^{2}=m_{\eta}^{2}$ to $q^{2}=-m_{\eta}^{2}$. Therefore, the mixing function is practically a constant. On the other hand, the intercept of our mixing function $(\beta)$ is fairly close to that obtained in chiral perturbation theory. Furthermore it should be noted that both in the GHT and in the PW approaches the $q^{2}$ variation of the $\pi-\eta$ mixing amplitude is much smaller than the one for the $\rho$ - $\omega$ mixing amplitude obtained in the same approaches [1] 3]. Finally, our mixing function gives an "on shell" value $\left(q^{2}=m_{\eta}^{2}\right)$ of $3800 \mathrm{MeV}^{2}$, which is slightly smaller (but within our estimated error) than that of the other two approaches and the one of $4200 \mathrm{MeV}^{2}$ obtained from the CSB NN force 17 19. 


\section{SUMMARY AND CONCLUSIONS}

In this paper, we use QCD sum rules to study $\pi-\eta$ mixing, choosing a combination of the two sum rules coming from the correlation functions of two axial vector currents. We are able to extract the leading behavior of the mixing function $\theta$ as a function of $q^{2}$ without the need to use four-quark condensates. We obtain $\theta\left(q^{2}=m_{n}^{2}\right)=3800 \mathrm{MeV}^{2}$, and a $q^{2}$-dependence of $\theta\left(q^{2}\right)$ that is compatible with zero. This is in contrast to the $\rho-\omega$ mixing amplitude, which varies strongly with $q^{2}$. Our results are in qualitative agreement with those obtained from various quark and hadronic models as well as from chiral perturbation theory. 


\section{REFERENCES}

[1] T. Goldman, J.A. Henderson, and A.W. Thomas, Few-Body Systems 12, 123 (1992).

[2] G. Krein, A.W. Thomas, and A.G. Williams, Phys. Lett. B317, 293 (1983).

[3] J. Piekarewicz and A.G. Williams, Phys. Rev. C 47, R2461 (1993).

[4] T. Hatsuda, E.M. Henley, Th. Meissner and G. Krein, Phys. Rev. C 49, 452 (1994).

[5] Kim Maltman, Los Alamos Report LA-UR-92-2549 (1992).

[6] Kim Maltman and T. Goldman, Los Alamos Report LA-UR-92-2910 (1992).

[7] J. Piekarewicz, Phys. Rev. C 48, 1555 (1993).

[8] E.M. Henley and G.A. Miller, in Mesons in Nuclei, eds. M. Rho and D.H. Wilkinson (North-Holland, Amsterdam, 1979).

[9] M.A. Shifman, A.I. Vainshtein and V.I. Zakharov, Nucl Phys. B147, 385, 448, 519 (1979).

[10] S. Narison, QCD Spectral Sum Rules (World Scientific, Singapore, 1990).

[11] L.J. Reinders, H. Rubinstein and S. Yazaki, Phys. Reports 127, No. 1, 1-97 (1985).

[12] M.A. Shifman, Vacuum Structure and QCD Sum Rules, ed. M.A. Shifman (NorthHolland, Amsterdam, 1992).

[13] R. Abegg, et al., Phys. Rev. Lett. 56, 2571 (1986); Phys. Rev. D 39, 2464 (1989).

[14] L.D. Knutson, et al., Nucl. Phys. A508, 185c (1990).

[15] S.A. Coon and M.D. Scadron, Phys. Rev. C26, 562 (1982); H.F. Jones and M.D. Scadron, Nucl. Phys. B155, 409 (1979).

[16] V.A. Novikov, M.A. Shifman, A.I. Vainstein, and V.I. Zakharov, Fortschr. Phys. 32, 60 (1984).

[17] P.C. McNamee, M.D. Scadron, and S.A. Coon, Nucl. Phys. A249, 483 (1975).

[18] S.A. Coon and M.D. Scadron, Phys. Rev. C 26, 562 (1982). 
[19] P.G. Blunden and M.J. Iqbal, Phys. Lett. B198, 14 (1987).

[20] J. Gasser and H. Leutwyler, Nucl. Phys. B250, 465, 517, 539 (1985). 


\section{FIGURES}

FIG. 1. The mixing parameters $g_{\pi}$ and $g_{\eta}$ as functions of the squared Borel mass $M^{2}$ 


\section{TABLES}

TABLE I. Comparisons of different approaches for the $\pi-\eta$ mixing function

\begin{tabular}{lcccc}
\hline \hline & $\alpha$ & $\beta\left(\mathrm{MeV}^{2}\right)$ & $\alpha / \beta\left(\mathrm{MeV}^{-2}\right)$ & $\theta\left(q^{2}=m_{\eta}^{2}\right)\left(\mathrm{MeV}^{2}\right)$ \\
\hline Chiral Perturbation & $1.08 \times 10^{-3}$ & 3808 & $2.836 \times 10^{-7}$ & 4131.68 \\
Theory [4,20] & & & & \\
\hline Hadronic Model [6] & $1.7 \times 10^{-3}$ & 3800 & $4.4 \times 10^{-7}$ & 4200 \\
\hline QCD Sum Rules & $3.5 \times 10^{-4}$ & 3700 & $9.45 \times 10^{-8}$ & 3800 \\
\hline \hline
\end{tabular}


This figure "fig1-1.png" is available in "png" format from: http://arxiv.org/ps/hep-ph/9403380v1 